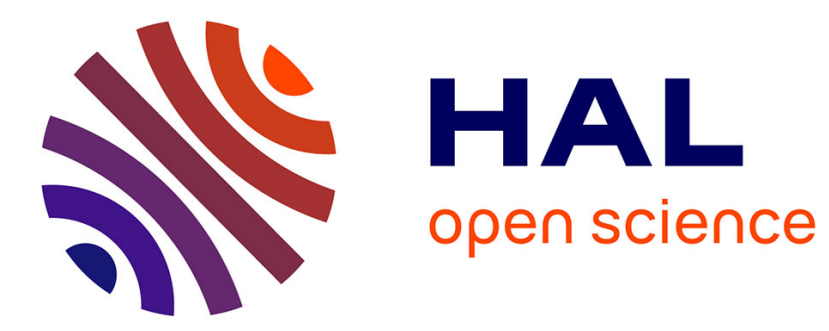

\title{
Eigenvalue spectra for Gaussian semilinear polymer graphs
}

H. Galina

\section{To cite this version:}

H. Galina. Eigenvalue spectra for Gaussian semilinear polymer graphs. Journal de Physique Lettres, 1984, 45 (22), pp.1077-1082. 10.1051/jphyslet:0198400450220107700 . jpa-00232452

\section{HAL Id: jpa-00232452 https://hal.science/jpa-00232452}

Submitted on 1 Jan 1984

HAL is a multi-disciplinary open access archive for the deposit and dissemination of scientific research documents, whether they are published or not. The documents may come from teaching and research institutions in France or abroad, or from public or private research centers.
L'archive ouverte pluridisciplinaire HAL, est destinée au dépôt et à la diffusion de documents scientifiques de niveau recherche, publiés ou non, émanant des établissements d'enseignement et de recherche français ou étrangers, des laboratoires publics ou privés. 
Classification

Physics Abstracts

$02.10-61.40 \mathrm{~K}$

\title{
Eigenvalue spectra for Gaussian semilinear polymer graphs
}

\author{
H. Galina \\ Institute of Organic and Polymer Technology, Technical University, \\ Wyb. Wyspiańskiego 27, 50-370 Wrocław, Poland
}

(Reçu le 8 juin 1984, révisé le 17 septembre, accepté le 27 septembre 1984)

\begin{abstract}
Résumé. - Les expressions des polynômes caractéristiques des matrices de Kirchhoff pour des graphes de polymères semilinéaires sont dérivées. On montre qu'une partie essentielle des valeurs propres, pour des graphes de cyclopolymères ou de polymères en peigne, peut se calculer comme racines de polynômes de Tchebychev. Ce spectre de valeurs propres des matrices de graphes de polymères semilinéaires est utile dans l'analyse des propriétés de configuration du modèle Gaussien de ces polymères.
\end{abstract}

\begin{abstract}
Expressions are derived for the characteristic polynomials of the Kirchhoff matrices of the graphs representing semilinear polymers. It is shown that a substantial part of the eigenvalue spectra for cyclopolymer as well as for comb-like polymer graphs can be calculated as the roots of the Chebyshev polynomials. The eigenvalue spectra of the semilinear polymer graph matrices are useful in the analysis of the configurational properties of the Gaussian models of these polymers.
\end{abstract}

The Gaussian model has an advantage over other methods of describing configurational properties of polymer molecules in that, it provides simple, often analytical, expressions for various experimentally verifiable configuration-dependent parameters [1]. In this model, the real polymer structure is usually replaced by a system of beads that represent atoms or groups of atoms and springs standing for chemical bonds [2, 3]. Thus, a polymer molecule can be considered as a molecular graph and the analysis of configurational properties can be reduced to the analysis of embeddings of its graph in a $d$-space [4]. General information on the Gaussian model, including approximations involved and applications, can be found, e.g., in the review by Eichinger [5].

Eichinger [4-6] has shown that the distribution of the square radius of gyration for a Gaussian molecule depends solely on the eigenvalue spectrum of the Kirchhoff matrix of its molecular graph. The Kirchhoff matrix of a graph of order $n$, called also the graph [7] or admittance [8] matrix, is the $n \times n$ symmetric matrix with the vertex degrees appearing in the main diagonal and with $(i, j)$-th entry being the negative of the number of edges linking vertex $i$ with vertex $j$.

Analytical expressions giving directly the eigenvalue spectra for linear (path), ring (cycle), star, and comb-like polymers, and polymer nets (regular graphs) are known [5,9]. In this Letter, considerably simplified forms of the characteristic polynomials of Kirchhoff matrices are derived for a general class of polymers referred to as the semilinear polymers which includes linear, comb-like, and cyclopolymers as the special cases of polymer molecules with an arbitrary structure appearing repeatedly along the path called the main chain. 
It is shown that the characteristic polynomials for cyclopolymers consist of products of Chebyshev polynomials or of combinations thereof, similarly, like for linear, star, ring, or comblike polymers [5]. In certain cases, the entire spectrum can be calculated without applying iteration procedures.

Let the characteristic polynomial of a symmetric matrix $\boldsymbol{M}$ be

$$
Q_{\mathbf{M}}(\hat{\lambda})=|\mathbf{M}-\hat{\lambda}|
$$

where $|$.$| denotes a determinant. Obviously, the nodes of Q_{M}$ are the eigenvalues of $\mathbf{M}$.

Consider a set of $m$ identical connected loopless graphs $G_{1}, G_{2}, \ldots, G_{m}=G$ each having two vertices denoted as in- and out-vertex, respectively. Let the out-vertex of $G_{1}$ be linked to the invertex of $G_{2}$, the out-vertex of $G_{2}$ be linked to the in-vertex of $G_{3}$, etc. Thus, each $G_{i}$ constitutes a repeat unit of a semilinear polymer, $\mathrm{H}_{m}$. If $\mathrm{G}$ has the order of $n$, the characteristic polynomial $Q_{K}(\hat{\lambda})$ of the Kirchhoff matrix $\mathbf{K}_{\mathbf{H}_{m}}$ of $\mathbf{H}_{m}$ is of order $m n$. The following application of the EichingerMartin algorithm $[5,10]$ splits $Q_{K}$ into a product of polynomials of orders $n-2$ and $2 m$.

Label in- and out-vertices of $\mathrm{G}_{i}$ with $2 i-1$ and $2 i$, respectively, and then the remaining vertices in each $\mathrm{G}_{i}$ with the numbers $2 m+(n-2)(i-1)+1$ through to $2 m+(n-2) i$, preferably along paths joining the in- and out-vertices in $\mathrm{G}_{i}$. Then,

$$
K=\left[\begin{array}{ll}
F & C^{\mathrm{T}} \\
\mathbf{C} & A
\end{array}\right]
$$

where $(2 m) \times(2 m)$ matrix

$$
\mathbf{F}=\left[\begin{array}{cccccc}
\delta_{\text {in }} & 0 & 0 & 0 & \ldots & 0 \\
0 & \delta_{\text {out }}+1 & -1 & 0 & \ldots & 0 \\
0 & -1 & \delta_{\text {in }}+1 & 0 & \ldots & 0 \\
0 & 0 & 0 & \delta_{\text {out }}+1 & \ldots & 0 \\
\ldots \ldots \ldots \ldots \ldots \ldots \ldots \ldots \ldots \ldots \ldots \ldots \ldots & \ldots \ldots \ldots
\end{array}\right]
$$

( $\delta_{\text {in }}$ and $\delta_{\text {out }}$ are the degrees of the in- and out-vertices, respectively), and

$$
\mathbf{A}=\left[\begin{array}{ccccc}
\mathbf{Z} & \mathbf{0} & \mathbf{0} & \ldots & \mathbf{0} \\
\mathbf{0} & \mathbf{Z} & \mathbf{0} & \ldots & \mathbf{0} \\
\ldots & \ldots & \ldots & \ldots & \ldots \\
\mathbf{0} & \mathbf{0} & \mathbf{0} & \ldots & \mathbf{Z}
\end{array}\right]
$$

is $m \times m$ block matrix with $\mathbf{Z}$ being the $(n-2) \times(n-2)$ submatrix of the Kirchhoff matrix of $\mathrm{G}$ obtained by deleting those two rows and columns that correspond to in- and out-vertices. C is the $(n-2) \times(2 m)$ sparse matrix.

Subtraction of $\lambda \mathbf{l}$ from (2) and application of the formula for a determinant of a block matrix yields

$$
|\mathbf{K}-\hat{\lambda} \mathbf{I}|=|\mathbf{A}-\hat{\mathbf{I}}|\left|\mathbf{F}-\hat{\lambda} \mathbf{I}-\mathbf{C}^{\mathrm{T}}[\mathbf{A}-\lambda \mathbf{I}]^{-1} \mathbf{C}\right|
$$

or, in virtue of (4),

$$
Q_{\mathbf{K}}=\left(Q_{\mathbf{Z}}\right)^{m} Q_{\mathbf{F} *}
$$

where $\mathbf{F}^{*}=\mathbf{F}-\mathbf{C}^{\mathrm{T}}[\mathbf{A}-\lambda \mathbf{I}]^{-1} \mathbf{C}$ and the use of (1) has been made. 
It is not difficult to verify by direct construction that the structure of $\mathbf{C}$ is such that the product $\mathbf{C}^{1}[\mathbf{A}-\lambda \mathbf{~ I I ~}]^{-1} \mathbf{C}$ reduces to the $m \times m$ block matrix where all but the diagonal $2 \times 2$ blocks vanish

$$
\mathbf{C}^{\mathrm{T}}[\mathbf{A}-\lambda \boldsymbol{I}]^{-1} \mathbf{C}=\left[\begin{array}{rrrrr}
\mathbf{P} & \mathbf{0} & \mathbf{0} & \ldots & \mathbf{0} \\
\mathbf{0} & \mathbf{P} & \mathbf{0} & \ldots & \mathbf{0} \\
\ldots & \ldots & \ldots & \ldots & \ldots \\
\mathbf{0} & \mathbf{0} & \mathbf{0} & \ldots & \mathbf{P}
\end{array}\right]
$$

A simple way to evaluate the elements of

$$
\mathbf{P}=\left[\begin{array}{ll}
p_{\alpha \alpha} & p_{\alpha \beta} \\
p_{\beta \alpha} & p_{\beta \beta}
\end{array}\right]
$$

is the following. Label the vertices in $\mathrm{G}$ which remained after deleting the in- and out-vertices so that $l_{\text {in }}$ vertices linked directly to the in-vertex with $v_{1}, v_{2}, \ldots, v_{l_{\text {in }}}$ edges bear labels $\alpha_{1}, \alpha_{2}, \ldots, \alpha_{l_{\text {in }}}$ respectively, and the $l_{\text {out }}$ vertices linked to the out-vertex with $\mu_{1}, \mu_{2}, \ldots, \mu_{\text {lout }}$ edges bear labels $\beta_{1}, \beta_{2}, \ldots, \beta_{l_{\text {out }}}$, respectively

$$
\left(\alpha_{i}, \beta_{i}=1,2, \ldots, n-2 ; v_{i}, \mu_{i}=1,2, \ldots ; \sum_{i}^{l_{\text {in }}} v_{i}=\delta_{\text {in }}, \sum_{i}^{l_{\text {out }}} \mu_{i}=\delta_{\text {out }}\right) .
$$

Then,

$$
\begin{aligned}
& p_{\alpha \alpha}=\sum_{i}^{l_{\text {in }}} \sum_{j}^{l_{\text {in }}} v_{i} v_{j} z^{\alpha_{i} \alpha_{j}} \\
& p_{\alpha \beta}=\sum_{i}^{l_{\text {in }}} \sum_{j}^{l_{\text {out }}} v_{i} \mu_{j} z^{\alpha_{i} \beta_{j}}=p_{\beta \alpha} \\
& p_{\beta \beta}=\sum_{i}^{l_{\text {out }}} \sum_{j}^{l_{\text {out }}} \mu_{i} \mu_{j} z^{\beta_{i} \beta_{j}}
\end{aligned}
$$

where $z^{q r}$ is the $(q, r)$-th element of $(\mathbf{Z}-\lambda \mathbf{I})^{-1}$.

For semilinear polymers, $\mathbf{G}$ is often symmetric, i.e., there exists a symmetry line cutting edges and/or vertices in $G$ so that it decomposes into two isomorphic subgraphs in which the in- and out-vertices remain mutual isomorphic images. In that case, $p_{\alpha \alpha}=p_{\beta \beta}\left(\right.$ also $\delta_{\text {in }}=\delta_{\text {out }}=\delta$ ), and $Q_{F^{*}}$ can be further simplified by noting that the $(2 \mathrm{~m}) \times(2 \mathrm{~m})$ determinant

$$
\begin{aligned}
\left|\mathbf{F}^{*}-\lambda\right| \mid & =u^{-2 m}\left|\begin{array}{cccccc}
v & -1 & 0 & 0 & \ldots & 0 \\
-1 & v+u & -u & 0 & \ldots & 0 \\
0 & -u & v+u & -1 & \ldots & 0 \\
\ldots \ldots \ldots & \ldots \ldots & \ldots \ldots & \ldots \ldots \\
0 & 0 & \ldots & -u & v+u & -1 \\
0 & 0 & \ldots & 0 & -1 & v
\end{array}\right| \\
& =2 u^{-m} y U_{m-1}[y+v]
\end{aligned}
$$

where $y(\lambda)=\left(v^{2}-1\right) / 2 u, v(\lambda)=\left(\delta-p_{\alpha \alpha}-\lambda\right) / p, u(\lambda)=1 / p_{\alpha \beta}$, and $U_{m-1}[$.$] is the (m-1)$-th order Chebyshev polynomial of the second kind. Equation (9) can be easily proven by induction. 
Let $\operatorname{cof}_{q r}|$.$| denote the cofactor of the (q, r)$-th element of a determinant. Since, by definition, $z^{q r}=\operatorname{cof}_{r q}|\mathbf{Z}-\lambda \mathbf{I}| /|\mathbf{Z}-\lambda \mathbf{I}|$, substitution of (Y) into (5a) yields

$$
Q_{\mathbf{K}}=2\left(\Sigma \Sigma v_{i} \mu_{j} \operatorname{cof}_{\beta_{i} \alpha_{j}}|\mathbf{Z}-\lambda \mathbf{I}|\right)^{m} y U_{m-1}[y+v]
$$

Equation (10) can be used for direct evaluation of eigenvalues of $\mathbf{K}_{\mathbf{H}_{m}}$; the task becomes relatively easy when the structure of symmetric repeat unit $\mathrm{G}$ is simple, and its order is not too high.

The origin of the elements in product (10) is easy to relate to the structure of the semilinear polymer graph. For exactly linear polymer, i.e., when $G$ is of order of 1 and consists simply of in- and out-vertices fused together into a single one, the characteristic polynomial as [5]

$$
Q_{\mathbf{K}}(\hat{\lambda})=-\lambda U_{m-1}[-\lambda / 2+1] .
$$

Similarly, the main chain of a semilinear polymer contributes to the characteristic polynomial with

$$
2 y U_{m-1}[y+v]
$$

where the zero eigenvalue is hidden in the front $y$ (there is only one zero eigenvalue in the spectrum of a connected graph Kirchhoff matrix). The argument of the Chebyshev polynomial $U_{m-1}$ is weighted with a combination of characteristic polynomials of Kirchhoff submatrices which correspond to the subgraphs pending from the main chain. The pending elements themselves contribute to equation (10) with a sum of characteristic polynomials of the signed minors of Kirchhoff submatrices of $G$ obtained by (i) deleting rows and columns corresponding to inand out-vertices, and, then, (ii) deleting one row (or column) and one column (or row) corresponding to the vertices adjacent to the in- and out-vertex, respectively.

The characteristic polynomial (10) assumes particularly simple form for cyclopolymers. The cyclopolymers are the progressions of identical cycles linked by single edges.

Let the two paths linking the in-vertex with the out- one in a unit of a cyclopolymer consist of $l$ and $k$ vertices, respectively. The substitution [10] $2-\lambda=2 x$ enables all minors of $\mathbf{Z}-\lambda \mathbf{I}$ to be expressed in terms of Chebyshev polynomials of the second kind [11]

$$
U_{i} \equiv U_{i}[x]=\sin [(i+1) \arccos x] / \sin [\arccos x] ;|x|<1 .
$$

Thus,

$$
\begin{gathered}
p_{\alpha \alpha}=\left(U_{k} U_{l-1}+U_{l} U_{k-1}\right) / U_{k} U_{l}, \\
p_{\alpha \beta}=1 / u=-\left(U_{k}+U_{l}\right) / U_{k} U_{l}, \\
v=-U_{k+l+1} /\left(U_{k}+U_{l}\right), \\
\Sigma \Sigma v_{i} \mu_{j} \operatorname{cof}_{\beta_{i} \alpha_{j}}|Z-\lambda|=-\left(U_{k}+U_{l}\right),
\end{gathered}
$$

etc.

Two extreme cases can be distinguished among cyclopolymers with distinct in- and outvertices : the symmetric cyclopolymers with both paths linking in- and out-vertex having the same length $(l=k$, Fig, 1a), and the spanning-path (Hamiltonian-path) cyclopolymers having a path that visits all its vertices $(l=0$, Fig. $1 b)$.

For the symmetric cyclopolymers, the characteristic polynomial reads

$$
Q_{K}(\hat{\lambda})=2^{m+1}\left(x^{2}-1\right)\left(U_{k}[x]\right)^{m+1} U_{m-1}\left[\left(x^{2}-1\right) U_{k}[x]+T_{k+1}[x]\right]
$$

where $T_{k+1}[x]=\cos [(k+1) \arccos x]$ is the Chebyshev polynomial of the first kind [11]. 


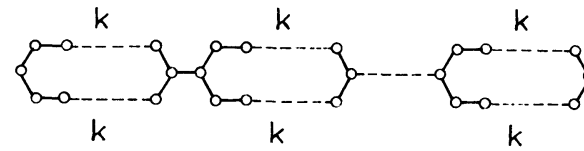

a

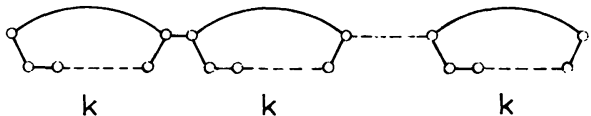

b

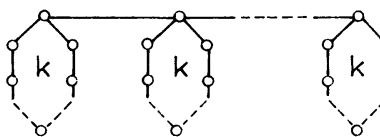

C

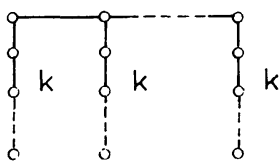

d

Fig. 1. - The graphs of cyclopolymers (a)-(c) and that of comb-like polymer (d) for which the Kirchhoff matrix characteristic polynomials are derived. For the cyclopolymers with distinct in- and out-vertices, two limiting cases are considered in detail. These are the symmetric cyclopolymers (a) and the spanningpath cyclopolymers (b). All semilinear polymers are considered to contain $m$ identical graphs (repeat unit) linked by single edges.

Equation (18) gives instantly $k(m+1)+2$ eigenvalues of the symmetric cyclopolymer Kirchhoff matrix since the nodes of $U_{k}$ are at

$$
\lambda_{i}=2-2 \cos [i \pi /(k+1)] ; \quad i=1,2, \ldots, k .
$$

For the spanning-path cyclopolymers, the characteristic polynomial is

$$
Q_{k}(\lambda)=2\left(U_{k}+1\right)^{m-1}\left(T_{k+2}-1\right) U_{m-1}\left[\left(T_{k+2}+U_{k+1}-1\right) /\left(U_{k}+1\right)\right] .
$$

Also equation (20) gives explicitly all but $(m-1)(k+1)$ eigenvalues.

For an even $k$, it can be rearranged into

$$
Q_{\mathbf{k}}=2^{m+1}\left(x^{2}-1\right)\left(U_{k / 2}\right)^{m+1}\left(T_{k / 2}\right)^{m-1} U_{m-1}\left[\left\{\left(x^{2}-1\right) U_{k / 2}+T_{k / 2+1}\right\} / T_{k / 2}\right]
$$

while for an odd $k$, into

$$
Q_{K}=2\left(w^{2}-1\right)\left(U_{k+1}[w]\right)^{m+1}\left(w^{-1} T_{k}[w]\right)^{m-1} U_{m-1}\left[\left\{2 w\left(w^{2}-1\right) U_{k+1}+T_{k+2}\right\} / T_{k}\right]
$$

where $w^{2}=1-\lambda / 4$. The first two terms in products (20a) and (20b) give the eigenvalues explicitly, including the $(m+1)$-fold ones, i.e., the zeros of $U_{k / 2}[x]$ and $U_{k+1}[w]$, respectively. The roots of $T_{k / 2}$ and $w^{-1} T_{k}$ correspond to the singularities of $U_{m-1}$ in (20a) and (20b), respectively, and must not be taken into account. The polynomials the nodes of which are the eigenvalues corresponding to the zeros of $U_{m-1}[$.$] in equation (18) and (20) are listed in table I for several k$ values.

For the comb-like molecules and for the cyclopolymers with just one vertex in the cycle contri- 
Table I. - The coefficients in the polynomials: $b_{0}+b_{1} x+b_{2} x^{2}+\cdots$, of which the nodes are the zeros of $U_{m-1}[$.$] in Eqs. (18) and (20) \left(a_{i}=\cos [i \pi / m] ; i=1,2, \ldots, m-1\right)$.

\begin{tabular}{|l|c|c|c|r|r|r|r|r|r|}
\hline & $k$ & $b_{0}$ & $b_{1}$ & $b_{2}$ & $b_{3}$ & $b_{4}$ & $b_{5}$ & $b_{6}$ & $b_{7}$ \\
\hline & 1 & $-1-a_{i}$ & -2 & 2 & 2 & & & & \\
Eq. (18) & 2 & $1-a_{i}$ & -3 & -5 & 4 & 4 & & & \\
$x=1-\lambda / 2$ & 3 & $1-a_{i}$ & 4 & -8 & -12 & 8 & 8 & & \\
& 4 & $-1-a_{i}$ & 5 & 13 & -20 & -28 & 16 & 16 & \\
& 5 & $-1-a_{i}$ & -6 & 18 & 38 & -48 & -64 & 32 & 32 \\
Eq. (20b) & 1 & $-1-a_{i}$ & -6 & 8 & & & & & \\
$x=1-\lambda / 4$ & 3 & $3+3 a_{i}$ & $6-4 a_{i}$ & -40 & 32 & & & & \\
& 5 & $-5-5 a_{i}$ & $6+20 a_{i}$ & $96+16 a_{i}$ & -224 & 128 & & & \\
\hline Eq. (20a) & 2 & -1 & $-2-a_{i}$ & 2 & 2 & & & & \\
$x=1-\lambda / 2$ & 4 & $1+a_{i}$ & -3 & $-5-2 a_{i}$ & 4 & 4 & & & \\
\hline
\end{tabular}

buting to the main chain (in- and out-vertices fused together (cf. Fig. 1c), the characteristic polynomials are

and

$$
Q_{\mathrm{K}}(\lambda)=2(x-1) U_{k}\left(U_{k}-U_{k-1}\right)^{m-1} U_{m-1}\left[T_{k+1} /\left(U_{k}-U_{k-1}\right)\right]
$$

$$
Q_{\mathbf{K}}(\lambda)=2\left(T_{k+1}-1\right)\left(U_{k}\right)^{m-1} U_{m-1}\left[\left(U_{k}+T_{k+1}-1\right) / U_{k}\right],
$$

respectively. They can be derived by direct application of the Eichinger-Martin algorithm [10], without using equation (9), and are given here for the sake of completeness.

For $m=1$, equations (18), (20), and (22) all give the known result for a ring polymer with $k$ beads [5]

$$
Q_{\mathbf{K}}(\lambda)=-\lambda\left\{U_{k}\left[\arccos (1-\lambda / 4)^{1 / 2}\right]\right\}
$$

\section{References}

[1] Flory, P. J., Principles of Polymer Chemistry (Cornell Univ. Press, Ithaca) 1953.

[2] Rouse, P. E., J. Chem. Phys. 21 (1953) 1272.

[3]'Flory, P. J., Statistical Mechanics of Chain Molecules (Interscience, New York) 1969.

[4] Martin, J. E., Eichinger, B. E., J. Chem. Phys. 69 (1978) 4588.

[5] Eichinger, B. E., Macromolecules 13 (1980) 1.

[6] Eichinger, B. E., Macromolecules 5 (1972) 496.

[7] Essam, J. W., Fisher, M. E., Rev. Mod. Phys. 42 (1970) 272.

[8] Cvetković, D. M., Doob, M., SAChs, H., Spectra of Graphs (VEB Deutscher Verlag der Wissenschaften, Berlin) 1980.

[9] Šolc, K., Macromolecules 5 (1972) 705.

[10] Eichinger, B. E., Martin, J. E., J. Chem. Phys. 69 (1978) 4595.

[11] Magnus, W., Oberhettinger, F., Soni, R. P., Formulas and.Theorems for the Special Functions of Mathematical Physics (3rd Ed., Springer-Verlag, Berlin) 1966. 